\title{
Exogenous siderophore-mediated iron uptake in Pseudomonas aeruginosa: possible involvement of porin OprF in iron translocation
}

\author{
JEAN-MARIE MeYER* \\ Laboratoire de Microbiologie, Institut de Biologie Moléculaire et Cellulaire du Centre National de la Recherche \\ Scientifique, Université Louis Pasteur, 4 rue Blaise-Pascal, F-67000 Strasbourg, France
}

(Received 16 October 1991; revised 30 December 1991; accepted 20 January 1992)

\begin{abstract}
In addition to the two siderophores pyoverdine and pyochelin synthesized by Pseudomonas aeruginosa ATCC 15692 (strain PAO1), several siderophores produced by other bacteria or fungi, namely cepabactin, salicylic acid, desferriferrichrysin, desferriferricrocin, desferriferrioxamine $B$, desferriferrioxamine $E$ and coprogen, were able to promote iron uptake with variable efficiencies into this bacterium. For most of these siderophores, these results were consistent with the growth stimulation produced by the same compounds in a plate bioassay. Desferriferrichrome A, enterobactin and desferriferrirubin, however, did not promote iron uptake, although enterobactin and desferriferrirubin stimulated bacterial growth. These paradoxical data are discussed in view of siderophore-inducible iron uptake systems, as demonstrated recently for enterobactin. Among the strains tested, including the wild-type PAO1, the pyoverdine-less mutant PAO6606 and the two porin-mutants $P$. aeruginosa H636 (oprF:: $\Omega$ ) and P. aeruginosa H673 (oprD::Tn501), only for the porin-OprF mutant were fewer siderophores able to promote iron uptake compared to the other strains. Such results suggest that beside specific routes for iron uptake $P$. aeruginosa is also able to take up siderophore-liganded iron through $\mathrm{OprF}$.
\end{abstract}

\section{Introduction}

To fulfill their iron requirement, many micro-organisms have evolved specialized molecules named siderophores which, when excreted in the cell environment, efficiently solubilize iron due to their high binding capacities for this element (Neilands, 1981; Matzanke et al., 1989). The ferrisiderophore complex is then usually translocated through the cell membranes by a highly specific active transport system involving several specialized proteins, the best characterized being the iron-regulated outer-membrane proteins (IROMPs) which act as ferrisiderophore receptors (Neilands, 1982). Iron-starved $E s$ cherichia coli cells synthesize several such IROMPs, each recognizing usually one (ferri)siderophore, including the endogenous siderophore produced by this bacterium, enterobactin, as well as the iron complexes of desferriferrichrome, desferriferrichrysin, coprogen or rhodotorulic acid, siderophores synthesized by other micro-organisms (Braun et al., 1987). Other bacteria, besides $E$. coli, can

\footnotetext{
- Author for correspondence. Tel. 88416153 ; fax 886075 50; email MBIO@FRIBCP51.EARN/BITNET.

Abbreviation: EDDHA, ethylenediamine di(hydroxyphenylacetic acid).
}

acquire iron from exogenous ferrisiderophores. For example, Mycobacterium smegmatis incorporates iron liganded to rhodotorulic acid (Ratledge, 1987), while Erwinia herbicola efficiently incorporates iron complexed to enterobactin, coprogen and, to a lesser extent, desferriferrichrome in addition to its own siderophore, desferriferrioxamine E (Berner et al., 1988). Also, growth of some Haemophilus strains is stimulated by enterobactin (Williams et al., 1990), whereas this compound induces in Pseudomonas aeruginosa a new iron uptake system specific for ferrienterobactin (Poole et al., 1990). $P$. aeruginosa was also seen to recognize, together with its own (ferri)siderophore pyoverdine ${ }_{\mathrm{PAO}}$, the iron complexes of pyoverdines synthesized by other strains of fluorescent pseudomonads, such as pyoverdine ${ }_{\mathrm{Pch}}$ produced by $P$. chlororaphis ATCC 9446 and pyoverdine $_{\mathrm{Pf} 113525}$ produced by $P$. fluorescens ATCC 13525, thus demonstrating an exception to the general rule of strict specificity of recognition established for many different Pseudomonas species with regard to their respective pyoverdine-mediated iron uptake capabilities (Hohnadel \& Meyer, 1988).

It is of some interest to define the iron metabolism and especially the iron incorporation potentials that an opportunistic pathogen like $P$. aeruginosa may develop 
(Bozenhart \& Ruden, 1987). In this paper the potential of 13 exogenous siderophores and citrate to promote iron uptake in $P$. aeruginosa is described. In addition, the question of whether these siderophore-iron complexes utilize one of the known porin pathways, OprF (Nikaido \& Hancock, 1986; Woodruff \& Hancock, 1988) or OprD (Trias \& Nikaido, 1990), as opposed to specific siderophore receptors, is explored.

\section{Methods}

Bacterial strains. The bacterial strains used in this study were the wild-type strain $P$. aeruginosa ATCC 15692 (strain PAO1) and three mutants derived originally from this strain. The pyoverdine-less mutant PAO6606, obtained by UV-mutagenesis, has been characterized previously (Hohnadel et al., 1986). The two porin-mutants, strains $\mathrm{H} 636$ and $\mathrm{H} 673$, were kindly provided by R.E.W. Hancock (University of British Columbia, Canada). Strain H673 is a oprD::Tn501 mutant which lacks the outer-membrane protein D2 (H. Meadows \& R. E. W. Hancock, unpublished data), whereas strain $\mathrm{H} 636$ is a $o p r F:: \Omega$ mutant lacking the outer-membrane protein F (Woodruff \& Hancock, 1988).

Plate bioassays. The influence of siderophores on bacterial growth was studied by a disk diffusion method on solid media. Plate bioassays were performed on nutrient agar and on succinate-agar media supplemented or not with $100 \mu \mathrm{g} \mathrm{ml}^{-1}$ of purified ethylenediamine di(hydroxyphenylacetic acid) [EDDHA], as previously described (Hohnadel \& Meyer, 1988). Succinate-agar media were supplemented with $1 \mathrm{~mm}$-methionine for growth of the methionine auxotroph strain PAO6606. Cells harvested from an overnight culture in the corresponding liquid medium were washed twice with sterile saline and plated evenly with the help of sterile glass beads at a concentration of $10^{6}$ cells per plate. Sterile paper disks were impregnated with $20 \mu l$ of filtersterilized $1 \mathrm{~mm}$-siderophore solutions ( $3 \mathrm{mM}$ for siderophores forming a $3: 1$ complex with iron, i.e. cepabactin) and deposited at the surface of the inoculated agar, four to five different compounds being tested per plate. Triplicate independent assays were run for each strain and for the different siderophores tested. Bacterial growth was observed at various times during incubation of the plates at $37^{\circ} \mathrm{C}$, and stimulation effects were visualized by development of the bacterial lawn first around the impregnated paper disks. This occurred, approximately $6 \mathrm{~h}$ after plating on nutrient agar without EDDHA, but required an 18 to $24 \mathrm{~h}$ incubation for growth to be visible on succinate-agar medium supplemented with EDDHA. No attempts were made to quantify the growth stimulation effects of the siderophores tested since, due to differences in the solubility of these compounds in aqueous media (e.g. pyoverdines are highly soluble in water, whereas for preparation of 1 or $3 \mathrm{mM}$ solutions of cepabactin, enterobactin or pyochelin, methanol was required), the diameter of the stimulation zone measured around the disks may not correlate with the potency of the siderophores. To prevent any growth inhibitory effect due to methanol for compounds dissolved in this solvent, the impregnated disks were first air-dried before being deposited on the surface of the plates.

${ }^{59} \mathrm{Fe}$ incorporation. The protocol used has been described previously (Cornelis et al., 1989). Briefly, cells harvested from overnight cultures in succinate medium at $37^{\circ} \mathrm{C}(1 \mathrm{mM}$-methionine/succinate medium for strain PAO6606) were washed twice in sterile saline and suspended in uptake medium (succinate medium with the nitrogen source omitted) at $\mathrm{OD}_{600}=3.0$. Uptake assays were run in $250 \mathrm{ml}$ Erlenmeyer flasks maintained at $30^{\circ} \mathrm{C}$ in a shaking ( 100 r.p.m.) waterbath and containing $8 \mathrm{ml}$ of uptake medium and $1 \mathrm{ml}$ of bacterial suspension. After $10 \mathrm{~min}$, uptake was initiated by adding $1 \mathrm{ml}$ of a mixture consisting of $5 \mu \mathrm{l}$ of ${ }^{59} \mathrm{Fe}$ [commercial solution: chloride form, specific activity $20 \mathrm{mCi}$ $\mathrm{mg}^{-1}$ (740 MBq $\mathrm{mg}^{-1}$ ); CEA, France], $50 \mu \mathrm{l}$ of a $1 \mathrm{mM}$ (or $3 \mathrm{mM}$; see above) siderophore solution and $945 \mu \mathrm{l}$ of uptake medium added $15 \mathrm{~min}$ after mixing the two first solutions. Samples $(1 \mathrm{ml})$ of bacterial suspension were withdrawn at various times during the $15 \mathrm{~min}$ incubation period and filtered through Millipore filters $(0.22 \mu \mathrm{m})$. Filters were then washed twice with $2 \mathrm{ml}$ of uptake medium before being counted for radioactivity using a Beckmann Gammamatic $\mathbf{4 0 0 0}$ counter. Assays without cells were performed as controls for the full solubilization of iron by the siderophores during the $15 \mathrm{~min}$ incubation time. All assays were run in triplicate for each ligand tested and data are usually presented as means of the three experimental values.

Siderophores and chemicals. The siderophores tested in this study included ferrirubin, coprogen and ferrichrysin, kindly provided by $\mathrm{Dr}$ N. A. C. Curtis (ICI, UK) and ferricrocin and ferrichrome A, a gift from Dr G. Winkelmann (Tübingen University, Germany). All these compounds, supplied as iron complexes, were deferrated by a previously described method (Wiebe \& Winkelmann, 1975) before their use in plate bioassays or in uptake studies. The different pyoverdines tested, originally from $P$. aeruginosa $\mathrm{PAO} 1, P$. aeruginosa ATCC 27853, $P$. fluorescens ATCC 13525, $P$. fluorescens ATCC 17400, $P$. fluorescens CCM 2798 and $P$. chlororaphis ATCC 9446, were purified according to previously published methods (Hohnadel \& Meyer, 1988), as were other siderophores of Pseudomonas origin, including cepabactin from $P$. cepacia A TCC 25416 (Meyer et al., 1989), pyochelin isolated either from $P$. aeruginosa PAO1 or from $P$. cepacia ATCC 25416 (Meyer et al., 1989), and desferriferrioxamine E from $P$. stutzeri ATCC 27588 (Meyer \& Abdallah, 1980). Enterobactin from $E$. coli strain AN311, kindly provided by Dr I. G. Young (Canberra University, Australia), was purified according to Young \& Gibson (1979). Desferriferrioxamine B (Desferal; Ciba-Geigy, Switzerland), salicylic acid and citric acid were of commercial origin.

\section{Results}

\section{Cross-feeding assays}

As visualized by plate bioassays, many siderophores of various bacterial origins were able to promote growth of the wild-type $P$. aeruginosa strain PAO1 (Table 1). Some of them, i.e. the endogenous pyoverdine of $P$. aeruginosa PAO1 as well as the pyoverdines of $P$. fluorescens ATCC 13525 and $P$. chlororaphis ATCC 9446, resulted in a strong stimulatory effect as judged by the bacterial lawn which developed first around the respective siderophoreimpregnated paper disks. Incubation at $37^{\circ} \mathrm{C}$ for $2 \mathrm{~d}$ was necessary for bacterial growth on the entire surface of the EDDHA/succinate agar plates to be visible, whereas some growth was already visible around the disks after a $12 \mathrm{~h}$ incubation period and had reached its maximal intensity $12 \mathrm{~h}$ later. In contrast, bacterial growth in the immediate vicinity of disks impregnated with the pyoverdines of $P$. fluorescens ATCC $17400, P$. fluorescens CCM 2798 or $P$. aeruginosa ATCC 27853 required, like growth on the entire surface of the plates, $48 \mathrm{~h}$ to develop. The other siderophores tested, chemically unrelated to pyoverdines, resulted in various responses: 
Table 1. Growth stimulation of the different $P$. aeruginosa strains mediated by various siderophores

The siderophores were used in this study as the deferrated forms. Growth effects were observed after $24 \mathrm{~h}$ at $37^{\circ} \mathrm{C}$ on EDDHAsupplemented succinate-agar medium and are indicated as follows: + , growth stimulation; 0 , no stimulation; $+/-$, variable responses (slight stimulation or no stimulation) observed among the triplicate independent experiments performed.

\begin{tabular}{|c|c|c|c|c|}
\hline \multirow[b]{2}{*}{ Siderophore } & \multicolumn{4}{|c|}{ Growth stimulation of strain: } \\
\hline & PAOI & PAO6606 & H636 & H673 \\
\hline Ferricrocin & + & $+1-$ & 0 & 0 \\
\hline Ferrichrysin & + & + & + & + \\
\hline Ferrirubin & + & + & + & + \\
\hline Ferrioxamine E & + & + & + & + \\
\hline Ferrioxamine B & + & + & + & + \\
\hline Ferrichrome A & $\mathbf{0}$ & 0 & 0 & 0 \\
\hline Enterobactin & + & + & + & + \\
\hline Pyochelin & $+1-$ & 0 & $+1-$ & 0 \\
\hline Cepabactin & 0 & $\mathbf{0}$ & $+1-$ & 0 \\
\hline Pyoverdine $_{\mathrm{PAO}}$ & + & + & + & + \\
\hline Pyoverdine $\mathbf{P a 2 7 8 5 3}$ & 0 & 0 & 0 & $\mathbf{0}$ \\
\hline Pyoverdine $_{\mathrm{Pf1} 13525}$ & + & + & + & + \\
\hline Pyoverdine $_{\mathrm{Pch}}$ & + & + & + & + \\
\hline Pyoverdine $_{\mathrm{Pn} 17400}$ & 0 & 0 & 0 & 0 \\
\hline Pyoverdine $_{\mathrm{Pf12798}}$ & 0 & 0 & 0 & 0 \\
\hline
\end{tabular}

the deferrated forms of ferricrocin, ferrichrysin, ferrirubin, ferrioxamine $\mathrm{E}$ and ferrioxamine B slightly stimulated the growth of $P$. aeruginosa PAOl, since weak growth was visible around the respective disks after a $24 \mathrm{~h}$ incubation, whereas enterobactin resulted in a greater stimulatory effect, bacterial growth around the disk appearing earlier (about $18 \mathrm{~h}$ ) and being more developed after $24 \mathrm{~h}$ incubation compared to growth with the other compounds. Desferriferrichrome A, as well as cepabactin, did not promote growth, whereas pyochelin led to variable results with one negative and two very slightly positive growth responses in the three experiments performed.

As seen in Table 1 , only a few differences were observed with the other $P$. aeruginosa strains tested, when compared to the wild-type $P$. aeruginosa PAO1. The pyoverdine-deficient mutant PAO6606 responded to the different siderophores similarly to strain PAO1 with only two exceptions: desferriferricrocin resulted in very poor stimulation of growth to no growth, whereas pyochelin did not stimulate growth in any of the three assays performed. The two porin-mutants, strains $\mathrm{H} 636$ and $H 673$, also demonstrated only small changes in their respective responses to the siderophores compared to the two other strains, the main changes being that desferriferricrocin did not promote the growth of these two strains, whereas in one of three experiments, cepabactin was seen to promote the growth of strain H636 slightly.

\section{${ }^{59} \mathrm{Fe}$ uptake}

The 13 iron complexes tested in iron uptake experiments performed on the wild type strain $P$. aeruginosa PAO1 could be classified in four groups, depending on the iron uptake kinetics experimentally observed (Fig. 1). The first group contained siderophores which very efficiently promoted iron uptake, namely the endogenous $P$. aeruginosa $\mathrm{PAO} 1$ siderophore, pyoverdine $\mathrm{PAO}_{\mathrm{PA}}$, and cepabactin, the major siderophore of $P$. cepacia ATCC 25416. The second group included citrate, salicylic acid, desferriferrichrysin and pyochelin, only one of which, pyochelin, is synthesized by $P$. aeruginosa PAO1. These ligands promoted iron incorporation at, on average, $30 \%$ of the efficiency obtained with the first group of siderophores. The third group, represented by desferriferrioxamine $B$, desferriferrioxamine $\mathrm{E}$, desferriferricrocin and coprogen, demonstrated only a weak ability to promote iron uptake, at 10 to $15 \%$ of the efficiency of pyoverdine $\mathrm{PAO}_{\mathrm{PAO}}$. Finally, desferriferrichrome A, enterobactin and desferriferrirubin, constituting the fourth group, were unable to promote iron uptake in $P$. aeruginosa PAO1.

The same experiments, done with the pyoverdine-less mutant PAO6606, resulted in roughly similar grouping of the different siderophores tested with certain changes. As shown in Fig. 2, iron uptake efficiencies were significantly increased for cepabactin, pyochelin, salicylic acid and citrate. In contrast, the efficiency of pyoverdine-iron uptake was about $30 \%$ less. Desferriferrichrysin, together with the siderophores of the third group (desferriferrioxamines B and E, desferriferricrocin and coprogen), demonstrated a similar capacity to promote iron uptake in PAO6606 compared to PAO1, and desferriferrichrome $A$, enterobactin and desferriferrirubin were again ineffective.

Iron uptake with the porin OprD (= protein D2) mutant (strain H673, Fig. 3) followed a similar pattern to that observed with PAO1: enhanced uptake with desferriferrioxamine $B$, decreased uptake with desferriferrichrysin, and no uptake with desferriferrioxamine $E$ were the only major differences observed for this strain compared to its parent strain.

For the porin-OprF-deficient mutant, strain $\mathrm{H} 636$ (Fig. 4), fewer siderophores were able to promote iron incorporation as compared to the other strains. Cepabactin and pyoverdine ${ }_{\mathrm{PAO}}$ were the most efficient siderophores as for strain PAO1, and citrate, salicylic acid and pyochelin still promoted uptake at about $30 \%$ of the efficiency of cepabactin and pyoverdine $\mathrm{PAO}_{\mathrm{PAO}}$. However, with the exception of coprogen, which still demonstrated a weak capacity to promote iron incorporation, none of the other siderophores promoted iron uptake in strain H636. This last group included desferriferrirubin, entero- 


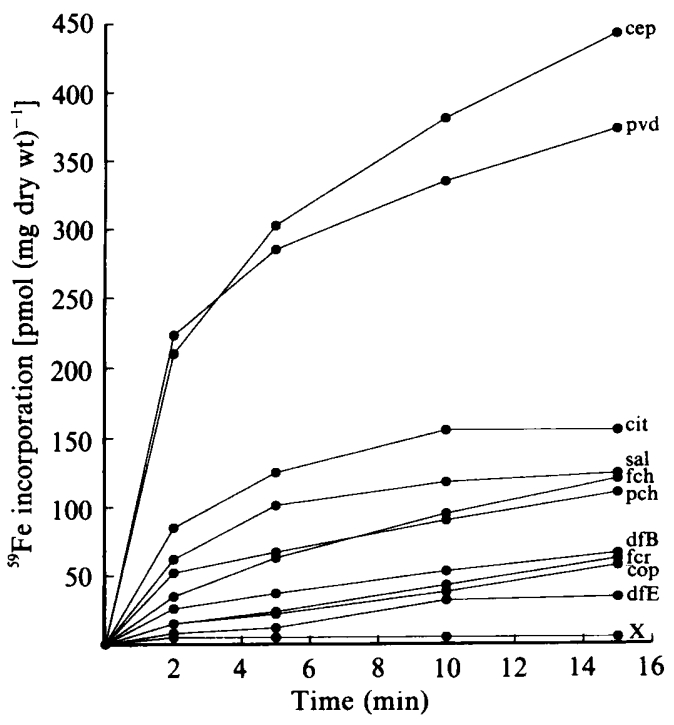

Fig. 1

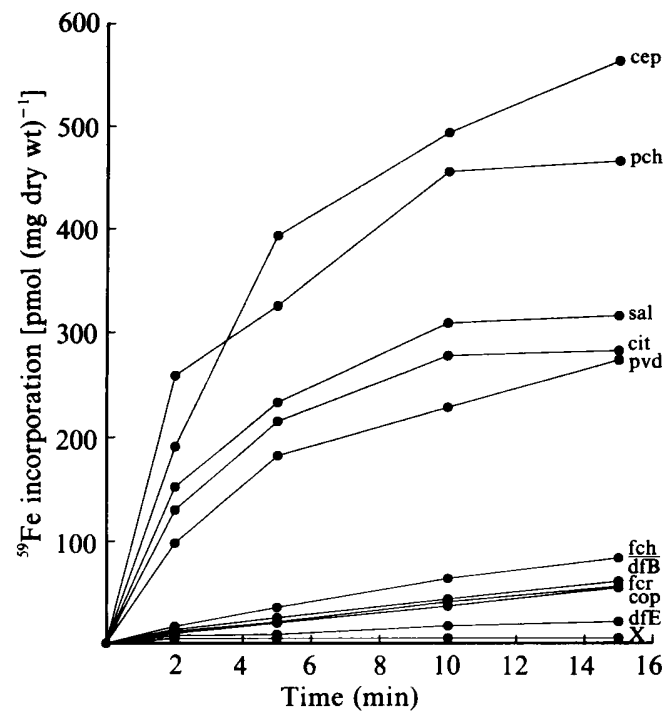

Fig. 2

Fig. 1. ${ }^{59} \mathrm{Fe}$ uptake mediated by various iron ligands in P. aeruginosa PAO1 (ATCC 15692). The ligands are abbreviated as follows: cep, cepabactin; pvd, pyoverdine ${ }_{\mathrm{PAO}}$; cit, citrate; sal, salicylic acid; fch, desferriferrichrysin, pch, pyochelin, dfB, desferriferrioxamine $B$; fcr, desferriferricrocin; cop, coprogen; dfE, desferriferrioxamine $E$. The line designated ' $X$ ' represents incorporation of ${ }^{59} \mathrm{Fe}$ liganded by desferriferrichrome $A$, enterobactin or desferriferrirubin.

Fig. 2. ${ }^{59} \mathrm{Fe}$ uptake mediated by various iron ligands in the pyoverdine-less mutant $P$. aeruginosa PAO6606. The ligands are abbreviated as in Fig. 1. The line designated ' $\mathrm{X}$ ' represents incorporation of ${ }^{59} \mathrm{Fe}$ liganded by desferriferrichrome A, enterobactin or desferriferrirubin.

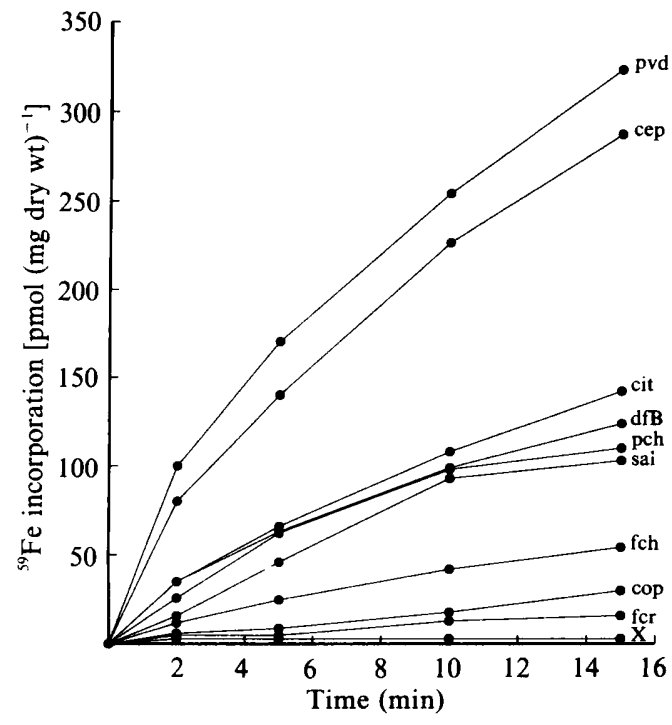

Fig. 3

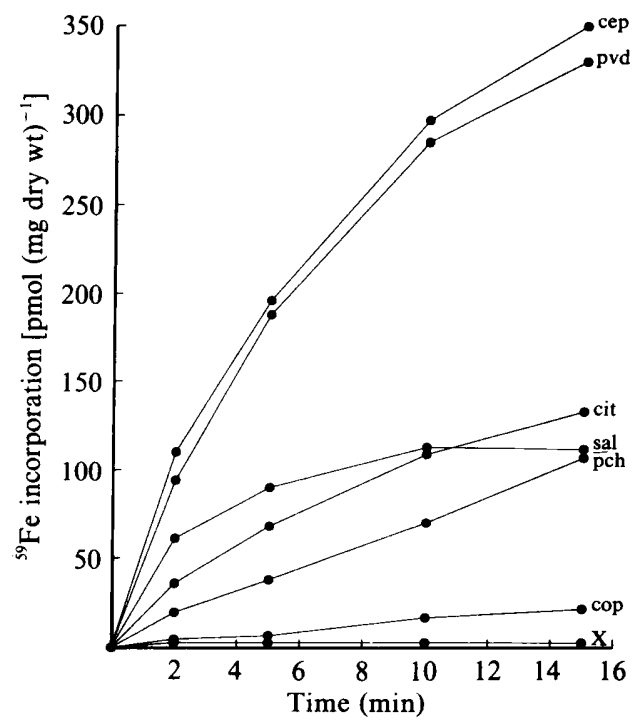

Fig. 4

Fig. 3. ${ }^{59} \mathrm{Fe}$ uptake mediated by various iron ligands in the porin OprD-deficient mutant $P$. aeruginosa H673. Abbreviations of the ligands are the same as in legend of Fig. 1. The line designated ' $X$ ' represents incorporation of ${ }^{59} \mathrm{Fe}$ liganded by desferriferrichrome $A$, enterobactin, desferriferrirubin or desferriferrioxamine $\mathrm{E}$.

Fig. 4. ${ }^{59} \mathrm{Fe}$ uptake mediated by various iron ligands in the porin-OprF-deficient mutant $P$. aeruginosa H636. The ligands are abbreviated as in Fig. 1. The line designated ' $\mathrm{X}$ ' represents incorporation of ${ }^{59} \mathrm{Fe}$ liganded by desferriferrichrome A, enterobactin, desferriferrirubin, desferriferrioxamine E, desferriferrioxamine B, desferriferricrocin or desferriferrichrysin. 
bactin and desferriferrichrome $A$, as for the other strains, but also desferriferrioxamine B, desferriferrioxamine $\mathrm{E}$, desferriferricrocin and desferriferrichrysin.

\section{Discussion}

From ${ }^{59} \mathrm{Fe}$ uptake studies involving 13 iron ligands mainly of microbial origin (siderophores) it can be concluded that $P$. aeruginosa ATCC 15692 (strain PAO1) is able to use the majority of the siderophores as an iron source. The only ones that were apparently not used by this bacterium were ferrichrome $\mathrm{A}$ and ferrirubin, two hydroxamate siderophores of fungal origin (van der Helm et al., 1987; Konetschny-Rapp et al., 1988), and enterobactin, the tricatecholate siderophore produced by several enterobacterial species, such as $E$. coli (O'Brien \& Gibson, 1970), Salmonella (Pollack \& Neilands, 1970) and Shigella (Payne, 1980).

Comparison of the uptake results with the growth stimulation properties of the siderophores towards the $P$. aeruginosa strains as visualized in plate bioassays (Table 1), indicated some discrepancies. For most of the siderophores, i.e. desferriferricrocin, desferriferrichrysin, desferriferrioxamine B, desferriferrioxamine $\mathbf{E}$ and pyoverdine $_{\mathrm{PAO}}$, both methods resulted in the same conclusion - that the compounds had a stimulatory effect. Moreover, desferriferrichrome A and the pyoverdines from $P$. aeruginosa ATCC 27853, $P$. fluorescens ATCC 17400 and $P$. fluorescens CCM 2798, which had already been seen to be inefficient in promoting iron uptake in $P$. aeruginosa PAO1 (Hohnadel \& Meyer, 1988; Cornelis et al., 1989), gave consistent results with both methods. Discrepancies, however, were found for desferriferrirubin and enterobactin, which promoted growth of all the strains tested, but were unable to promote iron uptake in these strains. In contrast, pyochelin and cepabactin, both efficient iron transporters as revealed by uptake assays, failed to promote bacterial growth in EDDHA-containing plate bioassays. Such results are not surprising considering the strong inhibitory effect of EDDHA on growth of exclusively cepabactin- and pyochelin-producing strains like PAO6606 (Hohnadel et al., 1986) or P. cepacia ATCC 25416 (Meyer et al., 1989), since these siderophores have a weaker affinity for iron than EDDHA.

As already discussed elsewhere (Hohnadel \& Meyer, 1988), results of plate cross-feeding experiments always need to be interpreted with caution since uncontrolled phenomena like competition for iron between the added siderophore and EDDHA, or with the endogenous siderophore excreted by the bacteria during growth, or toxicity of a given siderophore, may result in artifactual conclusions. Such undesired competition appears unlikely during uptake assays, done over a short period of time (15 min). Moreover, internal controls like the uptake results with strain PAO6606, a pyoverdine-deficient mutant, as well as the specific lack of iron incorporation with some siderophores for strain $\mathrm{H} 636$, attest to negligible iron exchange between siderophores during these assays.

Plate bioassays are still interesting, however, since they can reveal unsuspected features of siderophores, such as induction of specific iron transport pathways. K. Poole and colleagues have recently described the induction of an enterobactin-mediated iron uptake system by enterobactin itself in $P$. aeruginosa PAO1 (Poole et al., 1990). This inducibility of enterobactin may explain the discrepancy between the cross-feeding and uptake assays observed with this compound. In plate bioassays the growing bacteria were in contact with the inducer for a long period of time (18 to $24 \mathrm{~h}$ ), resulting in a growth stimulation effect likely to be due to induction of the enterobactin-mediated iron incorporation system, whereas this system was not induced during the uptake assays, since enterobactin and the non-proliferating cells were in contact for only a short period of time $(15 \mathrm{~min})$. It remains to be determined whether the desferriferrirubinrelated discrepancy results from such an induction, or is due to an iron exchange phenomenon.

The siderophores that promoted iron uptake by $P$. aeruginosa are of both bacterial and fungal origins, and belong to several different structural families. The fungal siderophores desferriferricrocin and desferriferrichrysin are structurally closely related to the inefficient siderophores desferriferrichrome A and desferriferrirubin (van der Helm et al., 1987; Konetschny-Rapp et al., 1988). Coprogen is a fungal trihydroxamate siderophore made from three $\partial N$-hydroxy- $\partial N$-acylornithine units (van der Helm et al., 1987). In addition, the present study included two desferriferrioxamine compounds of bacterial origin, desferriferrioxamine $B$ produced by actinomycetes (Bickel et al., 1960), and desferriferrioxamine E, produced by actinomycetes and also by some other bacterial species, such as Erwinia herbicola (Berner et al., 1988) and Pseudomonas stutzeri (Meyer \& Abdallah, 1980). The other effective siderophores are structurally unrelated to the siderophores cited above and included cepabactin, a pyridinone compound produced by the non-fluorescent Pseudomonas cepacia ATCC 25416 (Meyer et al., 1989), and salicylate, which is a precursor of pyochelin (Ankenbauer \& Cox, 1988), but also acts as a siderophore for a strain of Pseudomonas fluorescens (unpublished results). Together with these exogenous siderophores and the siderophores pyochelin and pyoverdine, produced by P. aeruginosa PAO1 itself (Cox \& Graham, 1979; Cox \& Adams, 1985), citrate was also able to mediate iron 
incorporation in this strain, a result in agreement with previous findings (Cox, 1980; Harding \& Royt, 1990).

Among the 10 iron-chelating compounds able to promote iron uptake in $P$. aeruginosa PAO1, two of them, pyoverdine $_{\mathrm{PAO}}$ and cepabactin, were particularly efficient compared with the others. For the wild-type strain PAOl, as well as for the two insertion mutants, H636 and H673, with altered porins, these two compounds led to an incorporation of iron that was two to three times greater than that observed with the next most efficient iron chelator, citrate.

It is not surprising that pyoverdine ${ }_{\mathrm{PAO}}$ was one of the most effective siderophores in promoting iron uptake since it is the endogenous siderophore of $P$. aeruginosa PAO1. The iron uptake efficiency for the second endogenous siderophore, pyochelin, was considerably lower, representing for strains PAO1, H636 and H673 less than $30 \%$ of the iron incorporation observed with pyoverdine. Such results support the conclusion that pyoverdine is the major siderophore of $P$. aeruginosa PAO1, in agreement with results on the comparative pathogenicity of pyoverdine-less or pyochelin-less mutants of $P$. aeruginosa PAOl (Ankenbauer et al., 1985). However, the present study demonstrates that, under appropriate circumstances, pyochelin can be more efficient than pyoverdine, as shown for the pyoverdineless mutant PAO6606. After $15 \mathrm{~min}$ incubation this strain had incorporated twice as much radioactivity as the other strains when iron was chelated by cepabactin, salicylate or citrate, whereas pyochelin-mediated iron incorporation was 4.5 times greater in this strain compared to pyochelin-mediated incorporation in the other strains. In contrast, using pyoverdine, iron was taken up with similar efficiency by all strains, including PAO6606. Therefore, in PAO6606, pyochelin supported nearly 2 -fold higher iron uptake than pyoverdine. From these results it seems that inability to synthesize pyoverdine results, in response to iron starvation, in increased efficiency of only some of the other possible iron acquisition routes, eg. cepabactin- or pyochelinmediated iron uptake. Previous studies concerning the phenotypic characterization of the mutant PAO6606 (Hohnadel et al., 1986) demonstrated that this mutation, affecting the biosynthesis of pyoverdine, had no apparent effect on the outer membrane proteins of this bacterium. Thus, the mechanism by which $P$. aeruginosa PAO6606 is able to increase the efficiency of its secondary iron uptake systems is apparently not related to an overexpression of IROMPs.

Among the siderophores which are not produced by $P$. aeruginosa but were effective in iron incorporation, cepabactin, as mentioned above, demonstrated an effectiveness equivalent to that of the major endogenous siderophore of $\boldsymbol{P}$. aeruginosa. Salicylate and citrate, which can be considered as siderophore-like compounds, were two other compounds which, regardless of the strain tested, resulted in a lesser but nevertheless consistent iron uptake efficiency. Cepabactin, 1-hydroxy5-methoxy-6-methyl-2(1H)-pyridinone (Meyer et al., 1989), has no chemical relationship with pyoverdine (Briskot et al., 1989) or pyochelin (Cox et al., 1981). Therefore, it appears likely that a special receptor may be involved at the level of the $P$. aeruginosa outer membrane for recognition of the 3:1 cepabactin-iron complex.

Under iron-starvation, $P$. aeruginosa strains usually express several IROMPs (Cornelis et al., 1987, 1989), the major one, a protein with an apparent molecular mass of $80 \mathrm{kDa}$ being the receptor for the pyoverdine-iron complex in strain PAO1 (Meyer et al., 1990). A search for mutants affected in the other IROMPs should clarify which protein could serve as the ferricepabactin receptor in the outer membrane of $P$. aeruginosa PAO1. A ferriccitrate receptor, corresponding to a $43 \mathrm{kDa}$ outermembrane protein, has recently been postulated in $P$. aeruginosa PAO1 (Harding \& Royt, 1990), suggesting the existence of a specific route for iron incorporation through this compound, similar to the FecA function in E. coli (Wagegg \& Braun, 1981). The receptor, if any, for iron-salicylate remains to be determined. Since this compound is a precursor and part of the structure of pyochelin, one could postulate that the $14 \mathrm{kDa}$ and/or the $75 \mathrm{kDa}$ IROMPs of $P$. aeruginosa may act as receptor(s) for both pyochelin- (Sokol \& Woods, 1983; Heinrichs et al., 1991) and salicylate-iron complexes.

The remaining exogenous siderophores able to promote iron uptake in $P$. aeruginosa, desferriferrioxamine $B$, desferriferrioxamine $\mathrm{E}$, desferriferrichrysin and desferriferricrocin, generally had a substantially lower efficiency compared to the other siderophores and, interestingly, were totally inefficient when tested with the strain H636. This strain has an insertion in the gene for the outer-membrane protein OprF (Woodruff \& Hancock, 1988), which has been shown to function as a porin, being a major uptake route across the outer membrane for hydrophilic compounds having a molecular mass less than $3000 \mathrm{Da}$ (Nikaido \& Hancock, 1986). This would suggest that ferrisiderophores may penetrate the bacterial cell through this pathway, and that the porin $\mathrm{OprF}$ is the only uptake route for iron complexes of desferriferrioxamine $B$, desferriferrioxamine $E$, desferriferrichrysin and desferriferricrocin. However, why ferrichrome A, ferrirubin and ferrienterobactin, which were shown to be inefficient for all the $\mathrm{OprF}^{+}$strains, were unable to use this route, remains to be explained.

The siderophores taken up by specific pathways involving IROMPs like pyoverdine or pyochelin in $P$. aeruginosa may also penetrate through porin channels. 
K. Poole and colleagues have recently isolated a $P$. aeruginosa mutant lacking the ferripyoverdine receptor (Poole et al., 1991). Interestingly, this mutant still presented a very low level of pyoverdine-mediated iron uptake, in a range of efficiency similar to that observed in the present study for desferriferrioxamine-, desferriferrichrysin- and desferriferricrocin-mediated iron uptake. The authors suggested the presence of a second uptake system for ferripyoverdine in $P$. aeruginosa which, in the light of results presented here, may be the porin pathway. More direct experimental evidence, such as permeability studies involving ferrisiderophores and porin-OprF proteoliposomes, are needed to assess this route of penetration for iron in $P$. aeruginosa.

In conclusion, this study underlines the particularly important role of iron in cellular metabolism by demonstrating that, beside the two endogenous systems involving pyoverdine and pyochelin and their respective receptors, $P$. aeruginosa is also able to fulfill its iron requirement by using exogenous siderophores and exogenous-siderophore-mediated iron uptake pathways, among them the porin-OprF channels. Another important feature revealed in this study is the increased capacity of iron uptake through secondary systems when the major route of iron incorporation becomes deficient. This unexpected mechanism of regulation remains to be explored.

I wish to express all my gratitude to G. Winkelmann, N. A. C. Curtis and I. G. Young for the gift of siderophores or strains. R. E. W. Hancock is particularly acknowledged for providing me with essential mutant strains and for the critical reviewing and improving of the manuscript. Excellent technical assistance was provided by Gérard Seyer.

\section{References}

ANKENBAUER, R. G. \& Cox, C. D. (1988). Isolation and characterization of Pseudomonas aeruginosa mutants requiring salicylic acid for pyochelin biosynthesis. Journal of Bacteriology 170, 5364-5367.

Ankenbauer, R., Sriyosachati, S. \& Cox, C. D. (1985). Effect of siderophores on the growth of Pseudomonas aeruginosa in human serum and transferrin. Infection and Immunity 49, 132-140.

Berner, I., Konetschny-Rapp, S., Jung, G. \& Winkelmann, G. (1988). Characterization of ferrioxamine $E$ as the principal siderophore of Erwinia herbicola (Enterobacter agglomerans). Biology of Metals 1, 51-56.

Bickel, H., Bosshardt, R., Gaumann, E., Reusser, P., Vischer, E., Voser, W., WetTstein, A. \& ZÄHNER, H. (1960). Stoffwechsel Produkte von Actinomyceten. Über die Isolierung und Charakterisierung der Ferrioxamine A-F, neuer Wuchstoffe der Sideramin Gruppe. Helvetica Chimica Acta 43, 2118-2128.

BOZENHART, K. \& RUDEN, H. (1987). Hospital infections caused by Pseudomonas aeruginosa. In Basic Research and Clinical Aspects of Pseudomonas aeruginosa, pp. 1-15. Edited by G. Döring, I. A. Holder \& K. Bozenhart. Basel: Karger.
Braun, V., HantKe, K., Eick-Helmerich, K., Köster, W., Pressler, U., SAUER, M., SCHÄFfer, S., SCHÖFfler, H., STAUdenMaIER, H. \& ZIMMERMANN, L. (1987). Iron transport systems in Escherichia coli. In Iron Transport in Microbes, Plants and Animals, pp. 35-51. Edited by G. Winkelmann, D. van der Helm \& J. B. Neilands. Weinheim: VCH Verlagsgesellschaft.

Briskot, G., Taraz, K. \& Budzikiewicz, H. (1989). Pyoverdin-type siderophores from Pseudomonas aeruginosa. Liebigs Annalen für Chemie, 375-384.

Cornelis, P., Hohnadel, D. \& Meyer, J. M. (1989). Evidence for different pyoverdine-mediated iron uptake systems among Pseudomonas aeruginosa strains. Infection and Immunity 57, 34913497.

Cornelis, P., Moguilevsky, N., Jaceues, J. F. \& Masson, P. L. (1987). Study of the siderophores and receptors in different clinical isolates of Pseudomonas aeruginosa. Antibiotic Chemotherapy 39, 290306.

Cox, C. D. (1980). Iron uptake with ferripyochelin and ferric citrate by Pseudomonas aeruginosa. Journal of Bacteriology 142, 581-587.

Cox, C. D. \& ADAMS, P. (1985). Siderophore activity of pyoverdin for Pseudomonas aeruginosa. Infection and Immunity 48, 130-138.

Cox, C. D. \& GraHAM, R. (1979). Isolation of an iron-binding compound from Pseudomonas aeruginosa. Journal of Bacteriology 137, 357-364.

Cox, C. D., Rinehart, K. L., Moore, M. L. \& Cook, J. C. (1981). Pyochelin: novel structure of an iron-chelating growth promoter for Pseudomonas aeruginosa Proceedings of the National Academy of Sciences of the United States of America 78, 4256-4260.

HARDING, R. A. \& ROYT, P. (1990). Acquisition of iron from citrate by Pseudomonas aeruginosa. Journal of General Microbiology 136, 18591867.

Heinrichs, D. E., Young, L. \& Poole, K. (1991). Pyochelin-mediated iron transport in Pseudomonas aeruginosa: involvement of a highmolecular-mass outer membrane protein. Infection and Immunity 59, 3680-3684.

van der Helm, D., Jalal, M. A. F. \& Hossain, M. B. (1987). The crystal structures, conformations and configurations of siderophores. In Iron Transport in Microbes, Plants and Animals. pp. 135-165. Edited by G. Winkelmann, D. van der Helm \& J. B. Neilands. Weinheim: VCH Verlagsgesellschaft

HOHNADEL, D. \& MEYER, J. M. (1988). Specificity of pyoverdinemediated iron uptake among fluorescent Pseudomonas strains. Journal of Bacteriology 170, 4865-4873.

Hohnadel, D., HaAs, D. \& MeYer, J. M. (1986). Mapping of mutations affecting pyoverdine production in Pseudomonas aeruginosa. FEMS Microbiology Letters 36, 195-199.

Konetschny-Rapp, S., HuschKa, H., WinkelmanN, G. \& Jung, G (1988). High performance liquid chromatography of siderophores from fungi. Biology of Metals 1, 9-17.

MatzanKe, B. F., Muller-Matzanke, G. \& Raymond, K. N. (1989). Siderophore-mediated iron transport. In Iron Carriers and Iron Proteins, pp. 1-121. Edited by T.M. Loehr. Weinheim: VCH Verlagsgesellschaft.

MEYeR, J. M. \& ABDallah, M. A. (1980). The siderochromes of nonfluorescent pseudomonads: production of nocardamine by Pseudomonas stutzeri. Journal of General Microbiology 118, 125-129.

Meyer, J. M., Hallé, F. \& Hohnadel, D. (1989). Cepabactin from Pseudomonas cepacia, a new type of siderophore. Journal of General Microbiology 135, 1479-1487.

MeYer, J. M., Hohnadel, D., KahN, A. \& CoRnelis, P. (1990). Pyoverdine-facilitated iron uptake in Pseudomonas aeruginosa: immunological characterization of the ferripyoverdine receptor. Molecular Microbiology 4, 1401-1405.

NeILANDS, J. B. (1981). Microbial iron compounds. Annual Review of Biochemistry 50, 715-731.

NeILANDS, J. B. (1982). Microbial envelope proteins related to iron. Annual Review of Microbiology 36, 285-309.

NikaIDO, H. \& HANCOCK, R. E. W. (1986). Outer membrane permeability of Pseudomonas aeruginosa. In The Bacteria: $A$ Treatise on Structure and Function, vol X, The Biology of Pseudomonas, pp. 145-193. Edited by J. R. Sokatch. New York: Academic Press. 
O'BriEn, I. G. \& Gibson, F. (1970). The structure of enterochelin and related 2,3-dihydroxy- $\mathrm{N}$-benzoylserine conjugates from Escherichia coli. Biochimica et Biophysica Acta 215, 393-402.

PAYNE, S. M. (1980). Synthesis and utilization of siderophores by Shigella flexneri. Journal of Bacteriology 143, 1420-1424.

Pollack, J. R. \& NeIlands, J. B. (1970). Enterobactin, an iron transport compound from Salmonella typhimurium. Eiochemical and Biophysical Research Communications 38, 989-992.

Poole, K., Neshat, S. \& Heinrich, D. (1991). Pyoverdine-mediated iron transport in Pseudomonas aeruginosa: involvement of a highmolecular-mass outer membrane protein. FEMS Microbiology Letters 78, 1-6.

PoOle, K., Young, L. \& Neshat, S. (1990). Enterobactin-mediated iron transport in Pseudomonas aeruginosa. Journal of Bacteriology 172, 6991-6996.

RATLEDGE, C. (1987). Iron metabolism in mycobacteria. In Iron Transport in Microbes, Plants and Animals, pp. 207-221. Edited by G. Winkelmann, D. van der Helm \& J. B. Neilands. Weinheim: VCH Verlagsgesellschaft.

Soxol, P. A. \& Woods, D. E. (1983). Demonstration of an ironsiderophore-binding protein in the outer membrane of Pseudomonas aeruginosa. Infection and Immunity 40, 665-669.
Trias, J. \& Niraido, H. (1990). Outer membrane protein $\mathrm{D}_{2}$ catalyzes facilitated diffusion of carbapenems and penems through the outer membrane of Pseudomonas aeruginosa. Antimicrobial Agents and Chemotherapy 34, 52-57.

WiEbe, C. \& WinkelmanN, G. (1975). Kinetics studies on the specificity of chelate iron uptake in Aspergillus. Journal of Bacteriology 123, 837-842.

WAGEGG, W. \& Braun, V. (1981). Ferric citrate transport in Escherichia coli requires outer membrane receptor protein FecA. Journal of Bacteriology 145, 156-163.

Willinms, P., Morton, D. I., Towner, K. J., Stevenson, P. \& GRIFFITHS, E. (1990). Utilization of enterobactin and other exogenous iron sources by Haemophilus influenzae, $H$. parainfluenzae and $H$. paraprophilus. Journal of General Microbiology 136, 2343-2350.

WOODRUFF, W. A. \& HANCOCK, R. E. W. (1988). Construction and characterization of Pseudomonas aeruginosa protein F-deficient mutants after in vitro and in vivo insertion mutagenesis of the cloned gene. Journal of Bacteriology 170, 2592-2598.

YounG, I. G. \& Gibson, F. (1979). Isolation of enterochelin from Escherichia coli. Methods in Enzymology 56, 394-398. 